\title{
Effective Field Theories for heavy probes in a hot QCD plasma and in the early universe
}

\author{
Miguel A. Escobedo1,2,a \\ ${ }^{1}$ Institut de Physique Théorique, Université Paris Saclay, CNRS, CEA, F-91191, Gif-sur-Yvette, France \\ ${ }^{2}$ Department of Physics, P.O. Box 35, 40014 University of Jyväskylä, Finland
}

\begin{abstract}
There are many interesting problems in heavy-ion collisions and in cosmology that involve the interaction of a heavy particle with a medium. An example is the dissociation of heavy quarkonium seen in heavy-ion collisions. This was believed to be due to the screening of chromoelectric fields that prevents the heavy quarks from binding, however in the last years several perturbative and lattice computations have pointed out to the possibility that dissociation is due to the finite lifetime of a quarkonium state inside the medium. Regarding cosmology, the study of the behavior of heavy Majorana neutrinos in a hot medium is important to understand if this model can explain the origin of dark matter and the baryon asymmetry. A very convenient way of studying these problems is with the use of non-relativistic effective field theories (EFTs), this allows to make the computations in a more systematic way by defining a more suitable power counting and making it more difficult to miss necessary resummations. In this proceedings I will review the most important results obtained by applying the EFT formalism to the study of quarkonium suppression and Majorana neutrinos, I will also discuss how combining an EFT called potential non-relativistic QCD (pNRQCD) with concepts coming from the field of open quantum systems it is possible to understand how the population of the different quarkonium states evolve with time inside a thermal medium.
\end{abstract}

\section{Introduction}

Although the energy and time scales are very different one can find very interesting similarities between the big bang and the little bangs that are created in Ultrarelativistic Heavy-Ion Collisions. Both systems start in a very hot and dense state and then they expand and cool down. During this expansion they go through various phases in which the relevant degrees of freedom are different. We can classify the different phases in the history of the universe and in the evolution of a heavy-ion collision (HIC) according to their temperature and in this sense we can say that their evolution is a thermal history.

Another similarity is that while we want to study the properties of systems that contain a large number of particles interacting with each other we can only detect them after the thermal medium does not exist anymore. To understand such type of environments it is useful to study systems that can act as probes of the medium, a good probe of the medium would be one that:

- Gives a clear signal that is easy to detect and difficult to confuse with another process.

\footnotetext{
ae-mail: miguel.a.escobedo-espinosa@jyu.fi
} 
Its properties are modified in a substantial way by the existence of the medium.

Examples of this kind of probes are jets in HICs and background radiation photons in cosmology. In this proceedings we are going to focus on heavy particles as probes of the medium. Some of the characteristics that make them good probes of the medium are the following:

- When they form bound states these are non-relativistic systems. This implies that they involve very separated energy scales and therefore they can probe the system at different energy and length scales.

- Their high mass will make their evolution clearly different to that of light particles, this will make them more likely to produce out of equilibrium phenomena which might be important.

The examples on which we are going to focus are:

- Heavy Majorana neutrinos in the early universe, which might have an important role in solving several problems in cosmology (see [1] and references therein).

- Heavy quarkonium in HIC, which were proposed as a signal of the formation of a quark-gluon plasma [2].

\subsection{Effective Field Theories for heavy particles}

We can call a particle heavy when its mass is much bigger than a number of other energy scales that appear in the problem at hand, therefore problems with heavy particles by definition always deal with very separated energy scales. It is well known that problems can arise when doing computations in quantum field theory in such cases. For example, if we have two very separated energy scales $\Lambda_{1}$ and $\Lambda_{2}$ such that $\Lambda_{1} \gg \Lambda_{2}$ and we try to do a naive perturbative expansion in the coupling constant we can find contributions that diverge as $\frac{\Lambda_{2}}{\Lambda_{1}} \rightarrow 0$. If this happens we can lose control of the perturbative expansion.

An efficient and systematic way of solving this kind of problems is by using Effective Field Theories (EFTs) to reorganize the computation [3]. An EFT is a quantum theory that gives the same results as a more general one (called the full theory) but that is only valid in a limited kinematic regime. The advantage is that the effects of the high energy degrees of freedom in the EFT are encoded in the coefficients that multiply the different operators in the Lagrangian and which are called Wilson coefficients, by looking at the dimensions of the operators one can reorganize the computation as an expansion on the ratio of the different energy scales. The example of the previous paragraph (heavy quark scattering) can be studied using the EFTs NRQCD [4, 5] (valid for energies smaller than the $M$ ) and pNRQCD [6, 7] (valid for energies smaller than $M v$ ), by doing so we can reorganize the perturbative computation of the scattering in a double expansion in $\alpha_{s}$ and $v$ and in this way we can be sure of not missing any needed resummation and we can take advantage of suppressions that are not always obvious in the full theory.

For the specific case we are interested here the use of EFTs have some additional advantages, the main reason is that they allow to skip some of the additional complications that thermal or nonequilibrium field theory have in comparison with "normal" quantum field theory. When dealing with a thermal or non-equilibrium medium the state of the system in only known in a probabilistic way, this implies that at a quantum level the description has to be done in terms of a density matrix instead of a vector state, the result of this in a field theory is a doubling of the degrees of freedom with respect to the vacuum case [8], however if we are in the case $M \gg T$ the impact of this doubling should be limited at least for the processes in which energies of order $M$ are involved. In the case $M \gg T$ (which is the one in which we focus in this work) the thermal medium does not affects the construction of the EFT so this part of the computation can be done at $T=0$. Moreover, computations in the EFT 
are simpler than in the full theory, for example a non-relativistic particle is described by a bispinor in QCD while in NRQCD it is just a spinor.

In the next sections we are going to discuss two specific problems in which the interaction of heavy particles with a medium is important and we are going to show how they can be efficiently studied using non-relativistic EFTs.

\section{Effective Field Theory for heavy Majorana particles in the early universe}

The model in which some heavy Majorana neutrinos are added to the standard model is a very interesting one (see [1] for a review) because it can potentially solve many problems of the standard model. The small mass of active neutrinos (whose order of magnitude can be inferred by neutrino oscillation experiments) can by explained by the seesaw mechanism [9] if one extends the standard model by introducing Majorana neutrinos. On top of that the inclusion of this particles breaks the lepton number conservation, this means that starting from a completely symmetric initial state (as for example the universe after inflation) it is possible to generate a lepton asymmetry that, through the sphaleron process, can later be translated into a baryon asymmetry [10]. Precisely the origin of the higher abundance of particles over antiparticles in the universe is one of the most important open problems in cosmology. Finally, the high mass and long mean free path of these Majorana neutrinos make them good dark matter candidates [11].

In order to determine, given a set of parameters, if sterile neutrino are a good dark matter candidate or if they can generate the needed lepton asymmetry for baryogenesis one needs to take into account their interaction with the high temperature medium that was the early universe. The computation of this can be very complicated, for example the generation of a non-zero lepton number involves the computation of high order diagrams in the perturbative expansion and it is a genuinely quantum process whose treatment in a hot environment is non trivial. Nevertheless, for reasons we have already explained in the introduction, we can expect that many simplifications will arise when considering heavy sterile neutrinos and the EFT formalism is the suitable tool to exploit them. In [12] we proposed to use an EFT for heavy Majorana particles (a very similar EFT was proposed in[13] ) to study their interaction with a thermal medium with a temperature much smaller than their mass. In the following we will discuss this Lagrangian for the case in which there is only one species of heavy Majorana neutrinos

$$
\mathcal{L}_{\mathrm{EFT}}=\mathcal{L}_{\mathrm{SM}}+\mathcal{L}_{\mathrm{N}}+\mathcal{L}_{\mathrm{N}-\mathrm{SM}},
$$

where $\mathcal{L}_{\mathrm{SM}}$ is just the standard model Lagrangian, $\mathcal{L}_{\mathrm{N}}$ is the part that only involves heavy Majorana neutrinos and has the form

$$
\mathcal{L}_{\mathrm{N}}=\bar{N}\left(i v \cdot \partial-\frac{i \Gamma_{T=0}^{(0)}}{2}\right) N+O\left(\frac{1}{M}\right),
$$

where $\Gamma_{T=0}^{(0)}$ is the decay width at zero temperature in the heavy-mass limit. All the possible interactions with other particles, and therefore the thermal effects, are due to the last term in eq. (1) which has the form

$$
\mathcal{L}_{\mathrm{N}-\mathrm{SM}}=\frac{1}{M} \mathcal{L}_{\mathrm{N}-\mathrm{SM}}^{(1)}+\frac{1}{M^{2}} \mathcal{L}_{\mathrm{N}-\mathrm{SM}}^{(2)}+\frac{1}{M^{3}} \mathcal{L}_{\mathrm{N}-\mathrm{SM}}^{(3)}+O\left(\frac{1}{M^{4}}\right)
$$

where $\mathcal{L}_{\mathrm{N}-\mathrm{SM}}^{(n)}$ includes all operators of dimension $4+n$. The fact that sterile neutrinos are not charged under any gauge group makes that all their interaction with low energy standard model particles (particles in the medium will have an energy much smaller than the mass of the sterile neutrinos) that respect the symmetries of the problem (for example Lorentz and gauge invariance) involves operators 
of dimension greater than 4 and therefore they are suppressed by inverse powers of the heavy neutrino mass $M$. The lowest dimension of such operators is

$$
\mathcal{L}_{\mathrm{N}-\mathrm{SM}}^{(1)}=a \bar{N} N \phi^{\dagger} \phi
$$

which involves the interaction with the Higgs particle represented by $\phi$.

The first application in [12] was to compute the thermal corrections to the decay width, this result was already known $[14,15]$ but we wanted to have a non-trivial cross-check of the applicability of this EFT. An advantage of using an EFT for this computation is that just by looking at the dimension of the operator we can already predict the size of the thermal corrections that it will produce, therefore if we just want the leading order thermal corrections in the limit $M \gg T$ it is enough to consider the term in eq. (4) as the influence of other terms will be suppressed by higher powers of $\frac{T}{M}$. The correction to the decay width coming from the term in eq. (4) will be of order $\frac{T^{2}}{M}$ and it will be only sensitive to the interaction of heavy Majorana neutrinos with low energy Higgs particles. By symmetry considerations it can be seen that it is impossible to construct a dimension 6 operator that would give a contribution in an isotropic and static plasma, therefore the next correction to the decay width in the heavy mass expansion will go like $\frac{T^{4}}{M^{3}}$ and will come from the term $\mathcal{L}_{\mathrm{N}-\mathrm{SM}}^{(3)}$ which has the form

$$
\begin{aligned}
& \mathcal{L}_{\mathrm{N}-\mathrm{SM}}^{(3)}=b \bar{N} N\left(v \cdot D \phi^{\dagger}\right)(v \cdot D \phi) \\
& +c_{1}^{f f^{\prime}}\left[\left(\bar{N} P_{L} i v \cdot D L_{f}\right)\left(\bar{L}_{f} P_{R} N\right)\right. \\
& \left.+\left(\bar{N} P_{R} i v \cdot D L_{f^{\prime}}^{c}\right)\left(\bar{L}_{f}^{c} P_{L} N\right)\right] \\
& +c_{2}^{f f^{\prime}}\left[\left(\bar{N} P_{L} \gamma_{\mu} \gamma_{v} i v \cdot D L_{f}\right)\left(\bar{L}_{f^{\prime}} \gamma^{v} \gamma^{\mu} P_{R} N\right)\right. \\
& \left.+\left(\bar{N} P_{R} \gamma_{\mu} \gamma_{v} i v \cdot D L_{f^{\prime}}^{c}\right)\left(\bar{L}_{f}^{c} \gamma^{v} \gamma^{\mu} P_{L} N\right)\right] \\
& +c_{3} \bar{N} N\left(\bar{t} P_{L} v^{\mu} v^{v} \gamma_{\mu} i D_{v} t\right)+c_{4} \bar{N} N\left(\bar{Q} P_{R} v^{\mu} v^{v} \gamma_{\mu} i D_{v} Q\right) \\
& +c_{5} \bar{N} \gamma^{5} \gamma^{\mu} N\left(\bar{t} P_{L} v \cdot \gamma i D_{\mu} t\right)+c_{6} \bar{N} \gamma^{5} \gamma^{\mu} N\left(\bar{Q} P_{R} v \cdot \gamma i D_{\mu} Q\right) \\
& +c_{7} \bar{N} \gamma^{5} \gamma^{\mu} N\left(\bar{t} P_{L} \gamma_{\mu} i v \cdot D t\right)+c_{8} \bar{N} \gamma^{5} \gamma^{\mu} N\left(\bar{Q} P_{R} \gamma_{\mu} i v \cdot D Q\right) \\
& -d_{1} \bar{N} N v^{\mu} v_{v} W_{\alpha \mu}^{a} W^{a \alpha v}-d_{2} \bar{N} N v^{\mu} v_{v} F_{\alpha \mu} F^{\alpha v} \\
& +d_{3} \bar{N} N W_{\mu \nu}^{a} W^{a \mu \nu}+d_{4} \bar{N} N F_{\mu \nu} F^{\mu \nu} \text {. }
\end{aligned}
$$

The fields $W_{\mu \nu}^{a}$ and $F_{\mu \nu}$ are the field strength tensors of the SU(2) gauge fields, $A_{\mu}^{a}$, and U(1) gauge fields, $B_{\mu}$, respectively. For the operators multiplying $c_{1}^{f f^{\prime}}$ and $c_{2}^{f f^{\prime}}$ the $\mathrm{SU}(2)$ indices of $L_{f}$ and $\bar{L}_{f^{\prime}}$ (representing light leptons) are contracted with each other while their Lorentz indices are contracted with gamma matrices and Majorana fields. Finally $Q$ and $t$ are respectively the right-handed quark doublet and the left-handed top (we only consider the top quark because its Yukawa coupling is much bigger than that of the others quarks).

All the information that was needed in order to construct this Lagrangian comes from the symmetries of the problem and from the knowledge of which are the active degrees of freedom. The only missing piece of information is encoded in the constants that multiply the different operators, which are called Wilson coefficients. In order to determine them we need to do some computation in the full theory (for example a scattering matrix element) and to find the coefficients that will make the EFT reproduce the same result, this procedure is called matching. Because the Wilson coefficients only encode the physics that happen at the scale $M$ they can be determined doing a $T=0$ computation and this is a great advantage as computations in thermal field theory are much more complicated. The 
matching of the coefficients that are needed in order to determine the corrections to the decay width was done in [12] and the values are shown there.

Now that we know the form of the EFT Lagrangian we can use it to determine the corrections to the decay width. The first correction in the heavy mass expansion is given by

$$
\Gamma_{T, L O}=2 \frac{\operatorname{Im} a}{M}\left\langle\phi^{\dagger}(0) \phi(0)\right\rangle_{T}=-\frac{|F|^{2} \lambda T^{2}}{8 \pi M} .
$$

This coincides with the results on $[14,15]$. Note that while in these references the result was obtained after a two loop computation in thermal field theory by using an EFT we decomposed it in two simpler steps

- A one loop computation at $T=0$ to obtain $\operatorname{Im} a$.

- A one loop computation in thermal field theory to obtain $\left\langle\phi^{\dagger}(0) \phi(0)\right\rangle_{T}$.

Results of higher order in the heavy mass expansion can be obtained by including the terms in eq. (5)

$$
\Gamma_{T}=\frac{|F|^{2} M}{8 \pi}\left[-\lambda\left(\frac{T}{M}\right)^{2}+\frac{\lambda}{2} \frac{\vec{k}^{2} T^{2}}{M^{4}}-\frac{\pi^{2}}{80}\left(\frac{T}{M}\right)^{4}\left(3 g^{2}+g^{\prime 2}\right)-\frac{7 \pi^{2}}{60}\left(\frac{T}{M}\right)^{4}\left|\lambda_{t}\right|^{2}\right] .
$$

\subsection{The lepton asymmetry}

The EFT we have discussed is a very powerful tool to compute thermal corrections to any process involving the interaction of heavy Majorana neutrinos with a thermal medium. The computation of the corrections to the decay width was our way to cross-check the exactness of the EFT and we have seen the we correctly reproduce the results of full theory calculations in a simpler way. The next step is to apply this technique to quantities that were not yet known and whose computation using just the full theory would be prohibitively difficult, an example of this is the lepton asymmetry that was discussed in $[16,17]$. The lepton asymmetry is a quantity that measures how much more likely is the decay of a Majorana neutrino into leptons than to antileptons and is defined by

$$
\epsilon_{I}=\frac{\sum_{f} \Gamma\left(v_{R, I} \rightarrow \ell_{f}+X\right)-\Gamma\left(v_{R, I} \rightarrow \bar{\ell}_{f}+X\right)}{\sum_{f} \Gamma\left(v_{R, I} \rightarrow \ell_{f}+X\right)+\Gamma\left(v_{R, I} \rightarrow \bar{\ell}_{f}+X\right)} .
$$

The sum runs over the SM lepton flavours, $v_{R, I}$ stands for the $I$-th heavy right-handed neutrino species, $\ell_{f}$ is a SM lepton with flavour $f$ and $X$ stands for any other SM particle not carrying a lepton number. This is a quantity that can be used as an input parameter to determine (via a Boltzmann equation or similar techniques [18]) what would be the number of leptons produced by Majorana particles in the early universe, this lepton asymmetry combined with the sphaleron process can give rise to the baryon asymmetry observed nowadays.

The computation of eq. (8) at $T=0$ already involves a two loop computation [10], if we were to compute its thermal corrections without using EFT techniques it would involve a three loop computation in thermal field theory, which is probably out of reach. However using the previously discussed EFT (whose extension to several heavy Majorana species is trivial) we can compute the leading order thermal correction by performing:

- A $T=0$ two loop computation to determine corrections to the Wilson coefficients.

- A one loop computation in thermal field theory to determine the expectation value of the EFT operators. 
Moreover, if we are only interested in the leading order corrections in the heavy mass limit (corrections that go like $\frac{T^{2}}{M^{2}}$ ) then we only have to focus in following pieces of the Lagrangian

$$
\mathcal{L}_{\mathrm{EFT}}=\mathcal{L}_{\mathrm{SM}}+\bar{N}_{I}\left(i v \cdot \partial-\delta M_{I}\right) N_{I}+\frac{i \Gamma_{I J}^{T=0}}{2} \bar{N}_{I} N_{J}+\frac{a_{I J}}{M_{I}} \bar{N}_{I} N_{J} \phi^{\dagger} \phi+\ldots,
$$

where the dots stand for operators of dimension bigger than 5. In writing the previous equation we have chosen to use the basis in which neutrino masses are diagonal. As seen by inspection, this does not necessarily coincide with the basis in which the rest of operators are diagonal.

The contributions to eq. (8) can be divided into two types, direct and indirect. Qualitatively they can be distinguished because the indirect is the one that gets enhanced when the masses are degenerate, we are not going to comment many details about the indirect asymmetry, in [16] it is shown that the Wilson coefficients from the EFT needed to get this contribution are the non-diagonal elements of $\Gamma_{I J}^{T=0}$ and $a_{I J}$ and the matching computation needed to get them is very similar to the one that is needed to predict the thermal corrections to the decay width.

Regarding the correction to the direct lepton asymmetry, its thermal correction can be written in this way

$$
\delta \epsilon_{I}=\frac{4\left\langle\phi^{\dagger}(0) \phi(0)\right\rangle_{T}}{M_{I}\left(\Gamma_{I I}^{l}+\Gamma_{I I}^{\bar{l}}\right)^{2}}\left(\operatorname{Im} a_{I I}^{l} \Gamma_{I I}^{\bar{l}}-\operatorname{Im} a_{I I}^{\bar{l}} \Gamma_{I I}^{l}\right),
$$

where $\Gamma$ has to be understood as $\Gamma^{T=0}$ and the superscript $l$ or $\bar{l}$ means respectively decay into leptons or to antileptons (this information can be retained during the matching process by using the cutting rules). What simplifies the computation dramatically is that both $\Gamma^{T=0}$ and $\operatorname{Im} a$ can be computed without taking into account the complications of thermal field theory. The almost degenerate masses case was studied in [16], it was found

$$
\begin{aligned}
\epsilon_{1, \text { direct }}^{T} & =\frac{\operatorname{Im}\left[\left(F_{1}^{*} F_{2}\right)^{2}\right]}{8 \pi\left|F_{1}\right|^{2}}\left(\frac{T}{M}\right)^{2}\left\{\lambda\left[2-\ln 2+(1-3 \ln 2) \frac{\Delta}{M}\right]\right. \\
& \left.-\frac{g^{2}}{16}\left[2-\ln 2+(3-5 \ln 2) \frac{\Delta}{M}\right]-\frac{g^{\prime 2}}{48}\left[4-\ln 2+(1-5 \ln 2) \frac{\Delta}{M}\right]\right\},
\end{aligned}
$$

where $M=M_{1}$ and $\Lambda=M_{2}-M_{1}$. The hierarchical case $M_{2} \gg M_{1}$ was studied in [17] giving the result

$$
\epsilon_{T}=-\frac{3}{16 \pi} \frac{M_{1}}{M_{2}} \frac{\operatorname{Im}\left[\left(F_{1}^{*} F_{2}\right)^{2}\right]}{\left|F_{1}\right|^{2}}\left[\left(-\frac{5}{3} \lambda+\frac{2 g^{2}+g^{\prime 2}}{12}\right)\left(\frac{T}{M_{1}}\right)^{2}+\frac{7 \pi^{2}}{20}\left|\lambda_{t}\right|^{2}\left(\frac{T}{M_{1}}\right)^{4}\right]
$$

\section{Effective Field Theories for heavy quarkonium in heavy-ion collisions}

The suppression of heavy quarkonium as a signal of the formation of a quark-gluon plasma was first proposed by Matsui and Satz in [19], it was based on the idea that the screening of the potential will suppress the interaction between the quark and the antiquark and therefore they will not bind. In perturbation theory screening means that we go from a Coulomb-type potential to a Yukawa-type

$$
V(r) \propto-\alpha_{S} C_{F} \frac{e^{-m_{D} r}}{r},
$$

where $m_{D}$ is the Debye mass, as this mass gets larger the number of bound states that can be formed diminishes. 
The phenomenon of screening tells us that some of the bound states that exist in a vacuum can not exists in a medium above a given temperature, however it can also happen that even if the state still exits in a medium its lifetime can be smaller than that of the fireball formed in heavy-ion collisions. The decay width of the more bound quarkonium states in the vacuum is very long however this can change dramatically at finite temperature if new decay channels are open. The computation in [20] showed that the potential of quarkonium (defined as the Wilson loop in Minkowski space) has an imaginary part that is related to a medium induced decay width of quarkonium, this is order of magnitude bigger than what is found in the vacuum at can be very efficient in dissociating quarkonium.

There is a very important detail that we have neglected in the previous discussion. We have been talking about the potential of quarkonium in a medium but it is not obvious at all that the evolution of quarkonium fulfills a Schrödinger equation or that thermal modifications can be encoded in a modification of the potential. In fact there is a long discussion on what is the correct input from Lattice QCD in order to extract quarkonium potential, the free energy or the internal energy (see [21] for a recent discussion). In order to give a systematic answer different groups started to apply non-relativistic EFTs to this problem $[22,23]$ because a similar strategy was already, and successfully, applied in the vacuum to answer similar problems.

Let us review now the non-relativistic EFTs used in the vacuum. There are three energy scales relevant for the study of quarkonium. The scale of the heavy quark mass $M$, called the hard scale. The scale of the typical three-momentum or inverse of the typical radius $\frac{1}{r}$, called the soft scale. Finally the scale of the binding energy $E$ or ultrasoft scale.

- NRQCD [4, 5] is the theory that is obtained after integrating out the hard scale. Its degrees of freedom are (apart from gluons and light quarks) a field for heavy quarks and another for heavy antiquarks which are normal spinors instead of bispinors. This EFT is often use to compute quarkonium production cross-sections.

- pNRQCD $[6,7]$ is the theory obtained from NRQCD when the soft scale is also integrated out. In this theory the degrees of freedom are a quantum field for color singlets and another for color octets, the reason is that gluons with an ultrasoft energy can not longer distinguish the heavy quark and antiquark and they are only sensitive to the full color state of the combination. pNRQCD has been used to obtain predictions for the spectroscopy and the decays of quarkonium.

The question on the limits a potential model gets a very precise answer in the EFT formalism. The evolution of the singlet at leading order in the ratio of energy scales is given by a Schrödinger equation and the potential is just a Wilson coefficient of pNRQCD that as any coefficient in a quantum field theory is affected by renormalization. However in pNRQCD there are also higher order contributions, suppressed by a factor $(r E)^{2}$, which can not be described by a potential model and that represent the interaction of the singlet field with ultrasoft gluons.

In order to apply a similar program at finite temperature one has to recognize the energy scales induced by the medium, in a perturbative plasma we have the temperature $T$ and the Debye mass $m_{D} \sim g T$. There is yet another smaller scale (of order $g^{2} T$ ) that is non-perturbative even in the limit $g \rightarrow 0$ but we are not taking it into account in the present discussion. Different physical situations will arise depending on the relation of these energy scales with the scales of heavy quarkonium physics.

\subsection{The case $\frac{1}{r} \gg T \gg E \gg m_{D}$}

This case was studied in detail in [24]. In this temperature regime neither the hard nor the soft scales are affected by the temperature, so we can use as starting point the same pNRQCD Lagrangian as in the vacuum. This means that thermal effects are a perturbation. The first thermal corrections to the 
binding energy are of order $\alpha_{s} r T E$ and they include terms that can not be encoded in a potential which are similar to the Bethe logs that appear in the Lamb shift.

Regarding the decay width, it is dominated by a process called gluo-dissociation [25, 26], in this process the singlet field absorbs a gluon from the medium and turns into an octet. In this case it also happens that part of the corrections can not be encoded in a potential.

The results of [24] were compared with a lattice computation in [27] finding a reasonable agreement.

\subsection{The case $\frac{1}{r} \gg T \gg m_{D} \gg E$}

This case is conceptually quite similar to the previous one, the main difference are the following:

- Up to very small corrections compared to the leading order thermal effects all medium modifications can be encoded in potential because all thermal scales are bigger than the binding energy.

- The decay width is dominated by inelastic parton scattering [28], this is the process in which a singlet scatters with a parton in the medium (that can be either a gluon or a quark) and as a result an octet and a parton is obtained. This process is $\alpha_{s}$ suppressed with respect to gluo-dissociation, however at high temperatures the phase space for this process is much bigger. The EFT power counting correctly predicts that for $m_{D} \gg T$ this is the dominant process.

\subsection{The case $T \sim \frac{1}{r}$}

In this case it is still true that the hard scale is unaffected by the medium so the $T=0$ NRQCD Lagrangian can still be used as a starting point. However the matching between NRQCD and pNRQCD will be affected by the temperature. In consequence, the singlet potential will get thermal corrections. These corrections are suppressed by an additional power of $\alpha_{s}$ in perturbation theory but not by any ratio of energy scales as happens in the $\frac{1}{r} \gg T$ case, the physical reason is that now the particles in the medium can already resolve separately the quark from the antiquark.

The potential in this temperature regime (both real and imaginary part) was studied in the analogous case of muonic hydrogen [29] (that can be understood as an Abelian version of quarkonium). The cross-section and decay width in this regime for quarkonium was studied in [28].

\subsection{The case $T \gg \frac{1}{r} \sim m_{D}$}

In this case both the matching between QCD and NRQCD and between NRQCD and pNRQCD are affected by the medium. From the point of view of particles with an energy of order $T$ the heavy quark and antiquark are seen as very separated entities, for this reason the leading thermal modification to the NRQCD Lagrangian is just a mass redefinition that has very little phenomenological impact [29]. However the singlet potential gets a contribution from the scale $m_{D}$ that is of the same order as the vacuum part even in perturbation theory. Indeed the potential computed in EFT at this temperature regime $[22,23]$ coincide with the complex potential that was previously computed in [20].

\subsection{Towards a computation of quarkonium nuclear modification factor in Effective Field Theory}

Until now we have discussed mediums in thermal equilibrium and corrections to the binding energy and decay widths of quarkonium. These are very important quantities in order to understand if a 
quarkonium state will exists at a given temperature and to compare with lattice computations. However what is observed in experiments is the nuclear modification factor $R_{A A}$ which is a measure of how many bound states are observed in a heavy-ion collision compared to a proton-proton collision. To compute this we need to study the evolution with time of quarkonium in a non-equilibrium plasma.

The binding energy and the decay width can be computed by looking at the pole of the timeordered singlet propagator exactly in the same way as we would do it at $T=0$. However to compute the evolution of quarkonium in a non-equilibrium plasma we have to do something quite different. For example, to know about the color singlets states at a given time we have to look at the expectation value

$$
f_{s}(\mathbf{x}, \mathbf{y})=\operatorname{Tr}\left(\rho S^{\dagger}(\mathbf{x}) S(\mathbf{y})\right),
$$

where $S$ is the color singlet field that appears in the pNRQCD Lagrangian and $\mathbf{x}$ and $\mathbf{y}$ refer to the relative coordinate as for the time being we ignore the center of mass movement. The previous expectation value has a natural interpretation in the framework of open quantum systems as the projection of the heavy quark reduced density matrix (the one that is obtained after doing a partial trace over gluon and light quarks degrees of freedom) into the subspace in which there is at least a singlet. Open quantum systems where introduced in the topic of quarkonium suppression in [30] and is a formalism that is often used in other fields of physics as quantum optics and many-body quantum theory.

In the work in preparation [31] we focus on the case $\frac{1}{r} \gg T$. As we already mentioned, we can use the $T=0$ pNRQCD Lagrangian to study this situation. Using the pNRQCD equations we can compute the evolution of $f_{s}$

$$
\partial_{t} f_{s}=-i\left[H_{s}, f_{s}\right]-\frac{1}{2}\left\{\Gamma_{s}, f_{s}\right\}+\mathcal{F}\left(f_{o}\right),
$$

the different parts of the right hand side of this equation have the following meaning:

- The term $-i\left[H_{s}, f_{s}\right]$ represents the unitary evolution that does not modify the total number of singlets. $H_{s}$ consist in the $T=0$ Hamiltonian (kinetic term plus Coulomb potential) plus a thermal correction proportional to $r^{2}$.

- $-\frac{1}{2}\left\{\Gamma_{s}, f_{s}\right\}$ is the effect of the decay width whose details have already been commented in the previous subsections.

- $\mathcal{F}\left(f_{o}\right)$ is a term that creates singlets if there are octets in the medium. This term is related with part of the decay width of the octet in such a way that the total number of quarkonium states (singlets plus octets) is conserved.

Eq. (15) is not a closed equation, in order to solve it we also need information about the octets. $f_{o}$ is defined as

$$
f_{o}^{a b}(\mathbf{x}, \mathbf{y})=\operatorname{Tr}\left(\rho O^{\dagger, a}(\mathbf{x}) O^{b}(\mathbf{y})\right),
$$

and its evolution equation is

$$
\partial_{t} f_{o}=-i\left[H_{o}, f_{o}\right]-\frac{1}{2}\left\{\Gamma_{o}, f_{o}\right\}+\mathcal{F}_{1}\left(f_{s}\right)+\mathcal{F}_{2}\left(f_{o}\right) .
$$

The different terms of eq. (17) have a very similar physical interpretation as in eq. (15). A remarkable consequence of the symmetries that constrain the construction of pNRQCD is that all the thermal information in these two evolution equations can be encoded in the correlator of two chromo-electric fields.

The explicit form of eqs. (15) and (17) can be very complex but there is a specific temperature regime in which many simplifications are possible, this is $\frac{1}{r} \gg T \gg E$. This case is even simple in 
the strong coupling regime as long as one assumes that all energy scales induced by the medium are bigger than the binding energy. In this case one can combine $f_{s}$ and $f_{o}$ in single matrix $\rho$ which is diagonal by boxes and where one box corresponds to $f_{s}$ and another to $f_{o}$. This matrix fulfills the equation

$$
\partial_{t} \rho=-i[H(\gamma), \rho]+\sum_{k}\left(C_{k} \rho C_{k}^{\dagger}-\frac{1}{2}\left\{C_{k}^{\dagger} C_{k}, \rho\right\}\right) .
$$

This expression has the form of a Lindblad equation [32]. This appears under very general assumptions (trace conservation, Markovian evolution...). The operators $C_{k}$ are called collapse operators and this specific case they have the form

$$
C_{i}^{0}=\sqrt{\frac{\kappa(t)}{N_{c}^{2}-1}} r^{i}\left(\begin{array}{cc}
0 & 1 \\
\sqrt{N_{c}^{2}-1} & 0
\end{array}\right),
$$

for the transition between singlets and octets, and

$$
C_{i}^{1}=\sqrt{\frac{\left(N_{c}^{2}-4\right) \kappa(t)}{2\left(N_{c}^{2}-1\right)}} r^{i}\left(\begin{array}{ll}
0 & 0 \\
0 & 1
\end{array}\right),
$$

for the transition between different octets states. This structure has a simple physical interpretation as a singlet that absorbs or emits a gluon will always turn into an octet while an octet doing the same can either turn into a singlet or remain being an octet. The Hamiltonian that enters the Lindblad equation can be written as

$$
H=\left(\begin{array}{cc}
h_{s} & 0 \\
0 & h_{o}
\end{array}\right)+\frac{r^{2}}{2} \gamma(t)\left(\begin{array}{cc}
1 & 0 \\
0 & \frac{N_{c}^{2}-2}{2\left(N_{c}^{2}-1\right)}
\end{array}\right),
$$

where $h_{s}$ and $h_{o}$ are the corresponding $T=0$ Hamiltonians. In conclusion, in this temperature regime all the information that we need from the medium can be encoded in the functions $\gamma(t)$ and $\kappa(t)$. In fact $\kappa(t)$ is a quantity that is also important for heavy quark diffusion [33] and has recently be computed on the lattice [34]. In order to make some predictions we also need to give some initial conditions and some information about the thermal evolution of the medium. We assume that the system thermalizes suddenly at $t_{0}=0.6 \mathrm{fm}$ and afterwards it follows a Bjorken-type evolution [35]. For the initial conditions we assume that at $t=0$ both $f_{s}$ and $f_{o}$ are proportional to a Dirac delta in $\mathbf{r}$ because the production process happens at very small distances and that $\frac{f_{o}}{f_{s}}=\alpha_{s} \delta$ where $\delta$ is an unknown constant.

In table 1 we show the results for different values of $\kappa, \gamma$ and $\delta$. We have chosen not to look at the most central collisions in order to avoid not fulfilling the condition $\frac{1}{r} \gg T$. We use a temperature at $t_{0}$ of $425 \mathrm{MeV}$ for the $30-50 \%$ centrality case and of $304 \mathrm{MeV}$ for the $50-100 \%$ centrality case. The results on the table are also corrected for feed-down following the same procedure as in [36]. We see that from all the entries in the table the one that gets closer to what is observed in experiments [37] is the last one. The numerical computation has been performed using the library QuTip [38, 39].

\section{Conclusions}

We have seen that EFTs are very useful techniques to study the interaction of heavy particles with a thermal medium. In both cosmology and heavy ion collisions there are several problems in which this physics is important. One of the main advantages is that it allows to clearly and systematically separate the high energy scales that are not modified by the medium from the low energy ones which 
Table 1. Results for $R_{A A}$ for different values of $\kappa, \gamma$ and $\delta$.

\begin{tabular}{|c|c|c|c|c|c|c|c|c|}
\hline \multicolumn{2}{|c|}{} & \multicolumn{3}{|c|}{$50 \%$ centrality } & \multicolumn{3}{c|}{$100 \%$ centrality } \\
\hline$\frac{K}{T^{3}}$ & $\frac{\gamma}{T^{3}}$ & $\delta$ & $R_{A A}(1 S)$ & $\frac{R_{A A}(2 S)}{R_{A A}(1 S)}$ & $\frac{R_{A A}(3 S)}{R_{A A}(1 S)}$ & $R_{A A}(1 S)$ & $\frac{R_{A A}(2 S)}{R_{A A}(1 S)}$ & $\frac{R_{A A}(3 S)}{R_{A A}(1 S)}$ \\
\hline 2.5 & -7.2 & 1 & 0.0317 & 0.550 & 0.492 & 0.578 & 0.696 & 0.650 \\
25 & -7.2 & 1 & 0.000262 & 1.87 & 2.89 & 0.183 & 0.488 & 0.439 \\
0.25 & -7.2 & 1 & 0.0643 & 0.719 & 0.731 & 0.664 & 0.849 & 0.824 \\
2.5 & -72 & 1 & 1.42 & 1.53 & 5.53 & 0.0426 & 0.944 & 1.13 \\
2.5 & -0.72 & 1 & 0.204 & 0.165 & 0.138 & 0.799 & 0.604 & 0.555 \\
2.5 & -7.2 & 0.1 & 0.0316 & 0.550 & 0.490 & 0.578 & 0.696 & 0.650 \\
2.5 & -7.2 & 10 & 0.0317 & 0.550 & 0.505 & 0.579 & 0.695 & 0.650 \\
0.25 & -0.72 & 1 & 0.699 & 0.235 & 0.194 & 0.940 & 0.927 & 0.914 \\
\hline
\end{tabular}

are crucially affected. An example of how useful and powerful this method is can be seen in our discussion about heavy Majorana neutrinos in the early universe where we computed the thermal corrections to the direct lepton asymmetry, a result that would have been much more difficult to obtain without using EFTs.

Regarding the very interesting case of quarkonium suppression in a medium, we have seen that we have quite good understanding of the physics in thermal equilibrium. We can compute thermal corrections in perturbation theory, in some cases up to next-to-leading order, of the binding energy and the decay width. We have reported recent advances in understanding the evolution of quarkonium in an out of equilibrium medium and how this can be used to make prediction of the nuclear modification factor observed in experiments.

\section{Acknowledgements}

I want to acknowledge the collaboration of Nora Brambilla, Simone Biondini, Jacoppo Ghiglieri, Joan Soto and Antonio Vairo in several of the works discussed here. During the preparation of this talk and the proceedings I have been supported by the European Research Council under the Advanced Investigator Grant ERC-AD-267258 and by the Academy of Finland, project 303756.

\section{References}

[1] M. Drewes, Int. J. Mod. Phys. E22, 1330019 (2013), 1303.6912

[2] T. Matsui, H. Satz, Phys. Lett. B178, 416 (1986)

[3] S. Weinberg, Physica A96, 327 (1979)

[4] W.E. Caswell, G.P. Lepage, Phys. Lett. B167, 437 (1986)

[5] G.T. Bodwin, E. Braaten, G.P. Lepage, Phys. Rev. D51, 1125 (1995), [Erratum: Phys. Rev.D55,5853(1997)], hep-ph/9407339

[6] A. Pineda, J. Soto, Nucl. Phys. Proc. Suppl. 64, 428 (1998), hep-ph/9707481

[7] N. Brambilla, A. Pineda, J. Soto, A. Vairo, Nucl. Phys. B566, 275 (2000), hep-ph/9907240

[8] M.L. Bellac, Thermal Field Theory (Cambridge University Press, 2011), ISBN 9780511885068 , 9780521654777

[9] P. Minkowski, Phys. Lett. B67, 421 (1977)

[10] M. Fukugita, T. Yanagida, Phys. Lett. B174, 45 (1986) 
[11] L.M. Krauss, S. Nasri, M. Trodden, Phys. Rev. D67, 085002 (2003), hep-ph/0210389

[12] S. Biondini, N. Brambilla, M.A. Escobedo, A. Vairo, JHEP 12, 028 (2013), 1307. 7680

[13] K. Kopp, T. Okui, Phys. Rev. D84, 093007 (2011), 1108.2702

[14] A. Salvio, P. Lodone, A. Strumia, JHEP 08, 116 (2011), 1106. 2814

[15] M. Laine, Y. Schroder, JHEP 02, 068 (2012), 1112.1205

[16] S. Biondini, N. Brambilla, M.A. Escobedo, A. Vairo, JHEP 03, 191 (2016), [Erratum: JHEP08,072(2016)], 1511.02803

[17] S. Biondini, N. Brambilla, A. Vairo, JHEP 09, 126 (2016), 1608. 01979

[18] E.W. Kolb, S. Wolfram, Nucl. Phys. B172, 224 (1980), [Erratum: Nucl. Phys.B195,542(1982)]

[19] T. Matsui, H. Satz, Phys. Lett. B178, 416 (1986)

[20] M. Laine, O. Philipsen, P. Romatschke, M. Tassler, JHEP 03, 054 (2007), hep-ph/0611300

[21] H. Satz, Eur. Phys. J. C75, 193 (2015), 1501.03940

[22] M.A. Escobedo, J. Soto, Phys. Rev. A78, 032520 (2008), 0804. 0691

[23] N. Brambilla, J. Ghiglieri, A. Vairo, P. Petreczky, Phys. Rev. D78, 014017 (2008), 0804.0993

[24] N. Brambilla, M.A. Escobedo, J. Ghiglieri, J. Soto, A. Vairo, JHEP 09, 038 (2010), 1007 . 4156

[25] N. Brambilla, M.A. Escobedo, J. Ghiglieri, A. Vairo, JHEP 12, 116 (2011), 1109.5826

[26] D. Kharzeev, H. Satz, Phys. Lett. B334, 155 (1994), hep-ph/9405414

[27] G. Aarts, C. Allton, S. Kim, M.P. Lombardo, M.B. Oktay, S.M. Ryan, D.K. Sinclair, J.I. Skullerud, JHEP 11, 103 (2011), 1109. 4496

[28] N. Brambilla, M.A. Escobedo, J. Ghiglieri, A. Vairo, JHEP 05, 130 (2013), 1303.6097

[29] M.A. Escobedo, J. Soto, Phys. Rev. A82, 042506 (2010), 1008.0254

[30] Y. Akamatsu, A. Rothkopf, Phys. Rev. D85, 105011 (2012), 1110. 1203

[31] N. Brambilla, M.A. Escobedo, J. Soto, A. Vairo, preprint:TUM-EFT 55/14

[32] G. Lindblad, Commun. Math. Phys. 48, 119 (1976)

[33] J. Casalderrey-Solana, D. Teaney, Phys. Rev. D74, 085012 (2006), hep-ph/0605199

[34] A. Francis, O. Kaczmarek, M. Laine, T. Neuhaus, H. Ohno, Phys. Rev. D92, 116003 (2015), 1508.04543

[35] J.D. Bjorken, Phys. Rev. D27, 140 (1983)

[36] M. Strickland, D. Bazow, Nucl. Phys. A879, 25 (2012), 1112. 2761

[37] S. Chatrchyan et al. (CMS), Phys. Rev. Lett. 109, 222301 (2012), 1208.2826

[38] J. Johansson, P. Nation, F. Nori, Computer Physics Communications 184, 1234 (2013)

[39] J. Johansson, P. Nation, F. Nori, Computer Physics Communications 183, 1760 (2012) 